\title{
Pulse Breakdown Strengths of Liquid, Gel and Solid Insulating Materials Using Closely Spaced Spherical Electrodes
}

\author{
P.J. LEASK* \\ BAE Systems, Advanced Technology Centre, Filton, Bristol, United Kingdom
}

\begin{abstract}
In this paper an evaluation of a number of different insulating materials, under pulse breakdown conditions, is described. The experimental setup used an 8 stage Marx generator in order to generate a high potential difference (in the range $80-220 \mathrm{kV}$ ) between two spherical electrodes which were spaced $1.5-3.5 \mathrm{~mm}$ apart. The breakdown voltage of each of the materials was recorded and the data was then post-processed in order to determine the breakdown strength of each of the samples.
\end{abstract}

PACS numbers: 77.22.Jp, 52.80.Mg, 52.80.Wq

\section{Introduction}

The use of Shell Diala mineral oil as a liquid insulator is well established in the field of pulsed power. However, over the last few years there has been a drive for increasingly compact high voltage devices that are capable of operating at ever greater potentials. As the field stresses increase to higher and higher levels there is a requirement for insulators which have breakdown strengths that are greater than those of established substances. Because of this a short study was undertaken into some of the various insulating materials that are currently available in order to try to find a higher performance alternative to Shell Diala. For this investigation samples of the following substances were procured and tested against Shell Diala D mineral oil:

- Midel 7131 produced by M\&I materials, Manchester, UK.

- Tetra High Energy Storage Oil (THESO) produced by Tetra Corporation, Albuquerque, USA.

- Luminol produced by Petro-Canada, Canada.

- 561 Silicone Transformer Oil produced by Dow Corning.

- Silicone Dielectric Gel (RS409-5692) manufactured by RS Components Ltd.

- Sylgard 184 Potting Compound produced by Dow Corning.

\footnotetext{
* e-mail: peter.leask@baesystems.com
}

\section{Experimental procedure}

In order to investigate the various materials a bespoke test cell was used. The cell consisted of two spherical electrodes (HV and earth), with the high voltage applied by a Marx generator. The electrodes were made from two $7 / 16^{\prime \prime}$ diameter stainless steel ball bearings, separated by a small gap, which could be accurately adjusted using a micrometer. A polycarbonate outer section provided mechanical rigidity and also contained the material under test. The Marx was an 8 stage self-breaking design that was charged in around $2 \mathrm{~ms}$ by an ALE $50 \mathrm{kV}$ power supply. The Marx utilized nitrogen as the switching gas and was capable of providing $\approx 70-260 \mathrm{kV}$ at up to $100 \mathrm{~Hz}$ repetition rate. The form of the voltage pulse generated by this setup is shown in Fig. 1. A North Star PVM-5 probe [1] and capacitive divider were used to measure the voltage across the test cell. This method, along with careful setup, and an appropriate choice of the Marx op-

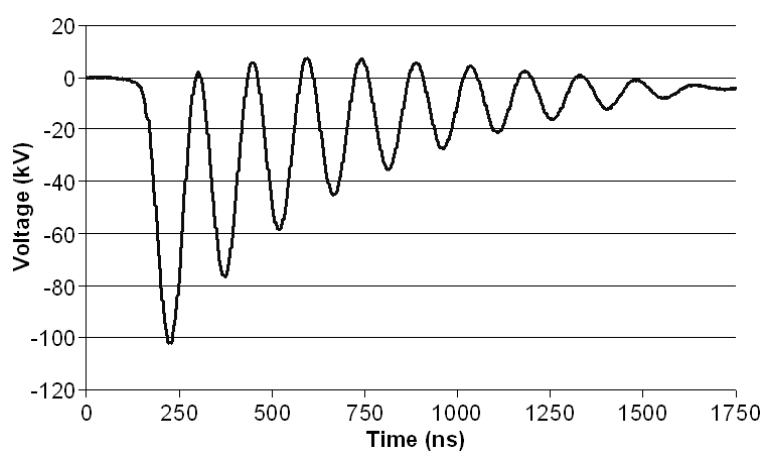

Fig. 1. Voltage pulse used for this experimental investigation. 
erating parameters allowed the output voltage to be accurately controlled, thus ensuring that any pulse variability between measurements taken at the same charge voltage was negligible. The Marx output voltage was monitored at all times to ensure that this was the case for every test.

For each of the measurements a fresh sample of the test substance was used and the gap between the two bearings was set accurately using the micrometer. The chamber was inserted into the circuit and the Marx was set to $80 \mathrm{kV}$ and run in duty cycle mode $(1 \mathrm{~s}$ at $100 \mathrm{~Hz}, 9 \mathrm{~s}$ off, for 300 pulses). If no breakdown was observed the output voltage was increased by $10 \mathrm{kV}$ and the test was re-run. Once a breakdown had been observed the test cell was removed, the balls and test material were replaced, and the chamber was placed back in the circuit, ready for the next test. For each gap spacing 2 repeat measurements were taken and the results were averaged.

\section{Results and discussion}

The collated breakdown voltage data, as a function of the gap distance, for each of the liquids, is shown in Fig. 2a. From these values it is possible to determine the breakdown strength (in $\mathrm{kV} / \mathrm{mm}$ ) of the various fluids. In doing this it is important to take into account the field enhancement provided by the electrode shape, which is itself a function of the gap distance. For equal spheres the field enhancement factor $(f)$ is given by

$$
f=\frac{\frac{D}{r}+1+\sqrt{\left(\frac{D}{r}+1\right)^{2}+8}}{4},
$$

where $D$ is the gap between the spheres and $r$ is their radius. The maximum electrical stress $\left(E_{\mathrm{m}}\right)$ is

$$
E_{\mathrm{m}}=V f / D,
$$

where $V$ is the applied voltage. If we apply these formulae to the measured breakdown voltage data then we obtain the chart shown in Fig. 2b. One might expect that, by taking into account the field enhancement factor, the calculated breakdown strength would be independent of the gap distance. This seems to be the case for the Shell Diala D and Midel 7131 oils, but is not true for the other liquids, which show a decrease in breakdown strength as the gap increases. The fluid values, as a function of distance, were all averaged in order to obtain a mean breakdown strength value for the range of gap distances measured in this study. The results, shown in Table, indicate that none of the liquids investigated has as high a breakdown strength as the Shell Diala D oil.

As well as the liquids that were investigated, a silicone gel and a solid potting compound were also evaluated. For these measurements the test cell was evacuated and then back filled, once full a vacuum was applied in order to remove air bubbles from the mixture and the material was then allowed to cure. For the silicone gel and the Sylgard 184 encapsulant breakdown data was only recorded with a $2.5 \mathrm{~mm}$ gap between the electrodes, due to the length of time required to process the mixtures. The

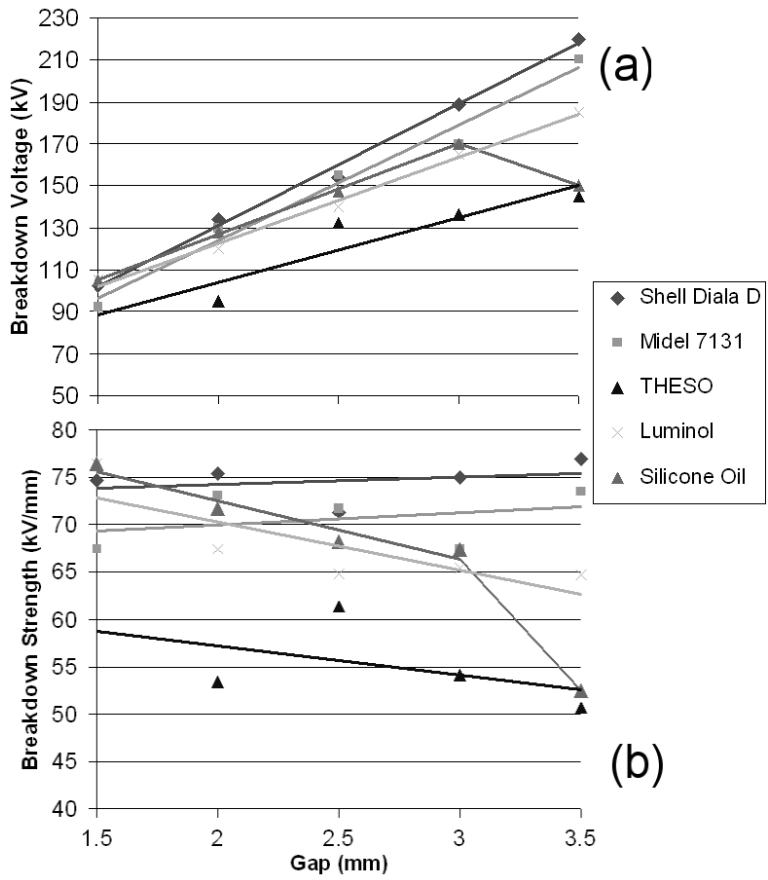

Fig. 2. (a) Breakdown voltage and (b) breakdown strength as a function of gap distance for each of the liquids under test.

TABLE

Average breakdown strengths for each of the materials under test.

\begin{tabular}{c|c|c}
\hline \hline Insulating fluid & $\begin{array}{c}\text { Breakdown strength } \\
{[\mathrm{kV} / \mathrm{mm}]}\end{array}$ & Permittivity \\
\hline Shell Diala D & 74.6 & 2.2 \\
Midel 7131 & 70.6 & 3.2 \\
Luminol & 67.8 & not available \\
Silicone Oil & 67.2 & 2.7 \\
THESO & 54.9 & 15.0 \\
Silicone Gel & 87.9 & not available \\
Sylgard 184 & 87.9 & 2.75
\end{tabular}

measurements were repeated twice and the results were then averaged (again the field enhancement was taken into account when analysing the data). As indicated in Table both the gel and the potting compound have significantly higher breakdown strengths than Shell Diala $\mathrm{D}$, although their use as direct replacements for a liquid insulator would be limited to cases where there was no need for regular access to the equipment under test.

In certain situations higher operating voltages may be realizable by using a combination of materials, as shown in Fig. 3. This diagram shows the electric field on a planar electrode (calculated using an electrostatics code), in the top plot only mineral oil is used as the insulating medium whereas in the bottom plot a combination of 

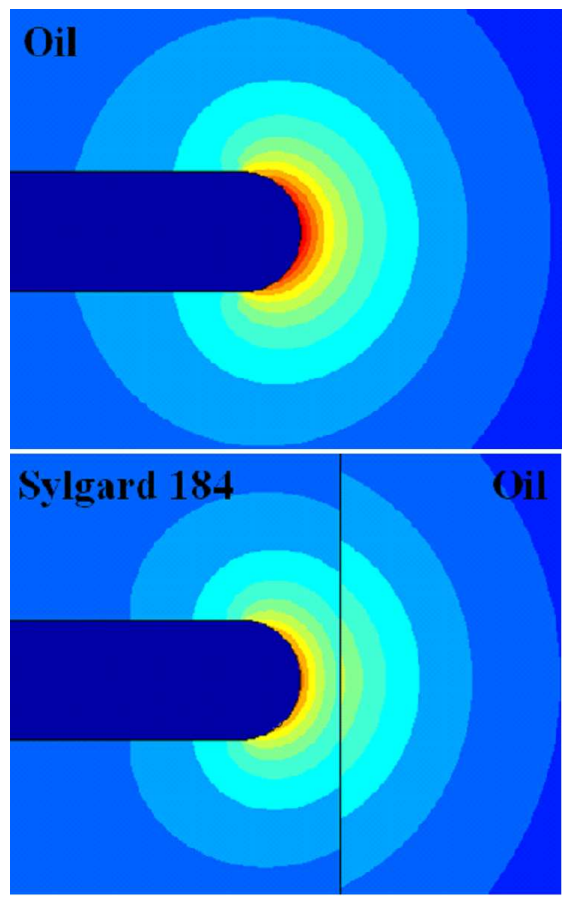

Fig. 3. Field on planar electrode with oil insulating medium (top) and oil and Sylgard 184 insulating media (bottom).

Sylgard 184 potting compound and oil is employed which lowers the field on the end of the electrode by $20 \%$. This technique, which is described in [2], works because the electric field in the layer with the highest permittivity is reduced, whereas in the lower permittivity region it is increased. This results in a more uniform distribution of the field in the gap between the electrodes.

\section{Conclusion}

The breakdown properties of a number of different dielectric materials have been measured using spherical electrodes spaced 1.5-3.5 mm apart. None of the candidate liquids investigated had as high a breakdown strength as Shell Diala D mineral oil, which remains the dielectric fluid of choice for pulsed power applications. However both the silicone gel and Sylgard 184 potting compound that were evaluated had significantly higher breakdown strengths than mineral oil and these materials may prove useful in certain applications. In addition it may be possible to reduce the electrical stress on key components, in certain scenarios, by employing combinations of materials.

\section{Acknowledgments}

This work was carried out as part of an Electronic Systems Research Programme. The author would like to thank the DTIC, MBDA and acknowledge the support of the MoD HPM Principal Programme.

\section{References}

[1] North Star High Voltage, Arizona (USA).

[2] S.M. Lebedev, O.S. Gefle, Yu.P Pokholkov, V.I. Chichikin, High Voltage Engineering Symp., IEEE 1999, p. 304. 